Article

\title{
Common Coincidence Points and Common Fixed Points in Fuzzy Semi-Metric Spaces
}

\author{
Hsien-Chung Wu \\ Department of Mathematics, National Kaohsiung Normal University, Kaohsiung 802, Taiwan; \\ hcwu@nknucc.nknu.edu.tw
}

Received: 26 January 2018; Accepted: 16 February 2018; Published: 23 February 2018

\begin{abstract}
We propose the so-called fuzzy semi-metric space in which the symmetric condition is not assumed to be satisfied. In this case, there are four kinds of triangle inequalities that should be considered. The purpose of this paper is to study the common coincidence points and common fixed points in the newly proposed fuzzy semi-metric spaces endowed with the so-called $\bowtie$-triangle inequality. The other three different kinds of triangle inequalities will be the future research, since they cannot be similarly investigated as the case of $\bowtie$-triangle inequality.
\end{abstract}

Keywords: common coincidence points; common fixed points; fuzzy semi-metric space

\section{Introduction}

The topic of probabilistic metric space has been studied for a long time. In order to realize the development and basic idea of probabilistic metric space, we may refer to Schweizer and Sklar [1-3], Hadžić and Pap [4] and Chang et al. [5]. The so-called Menger space is a special kind of probabilistic metric space. Using the similar ideas of Menger space, Kramosil and Michalek [6] proposed the fuzzy metric space as follows.

Let $X$ be a nonempty universal set, let $*$ be a t-norm, and let $M$ be a mapping defined on $X \times X \times[0, \infty)$ into $[0,1]$. The 3-tuple $(X, M, *)$ is called a fuzzy metric space if and only if the following conditions are satisfied:

- for any $x, y \in X, M(x, y, t)=1$ for all $t>0$ if and only if $x=y$;

- $M(x, y, 0)=0$ for all $x, y \in X$;

- $M(x, y, t)=M(y, x, t)$ for all $x, y \in X$ and $t \geq 0$;

- $M(x, y, t) * M(y, z, s) \leq M(x, z, t+s)$ for all $x, y, z \in X$ and $s, t \geq 0$ (the so-called triangle inequality).

Sometimes the mapping $M$ is called the fuzzy metric of the space $(X, M, *)$. The fuzzy metric $M$ in $(X, M, *)$ can also be regarded as a kind of membership function of a fuzzy subset of $X \times X \times[0, \infty)$. The first and second conditions of fuzzy metric space $(X, M, *)$ say that the fuzzy metric $M(x, y, t)$ can be interpreted as the membership degree of the distance that is less than or equal to $t$ between $x$ and $y$. In this paper, we shall not consider the symmetric condition $M(x, y, t)=M(y, x, t)$. Therefore, we shall consider the so-called fuzzy semi-metric space. Since the symmetric condition is not taken into account, we shall separately consider four kinds of triangle inequalities.

Singh and Chauhan [7], Vasuki [8] and Wang et al. [9] studied the common fixed points in fuzzy metric spaces. De la Sen et al. $[10,11]$ studied the so-called fuzzy best proximity points and fuzzy best proximity coincidence points. In this paper, using the four different kinds of triangle inequalities, we shall study the common fixed points in fuzzy semi-metric spaces. We also investigate the common coincidence points in fuzzy semi-metric spaces. Although the common fixed points 
are also the common coincidence points, regarding the uniqueness, the sufficient conditions will be completely different.

This paper is organized as follows. In Section 2, we propose the fuzzy semi-metric space that is endowed with four kinds of triangle inequalities. In Section 3, we introduce the auxiliary functions that will be used to study the Cauchy sequence in fuzzy semi-metric space. In Section 4, we study the Cauchy sequence in fuzzy semi-metric space by means of the auxiliary functions established in Section 3. In Section 5, we derive many kinds of common coincidence points in fuzzy semi-metric spaces that can be endowed with the different types of triangle inequalities introduced in Section 2. Finally, in Section 6, we also study the common fixed points in fuzzy semi-metric spaces.

\section{Fuzzy Semi-Metric Spaces}

We first introduce the concept of triangular norm that is simply called a t-norm. We say that the function $*:[0,1] \times[0,1] \rightarrow[0,1]$ is a t-norm if and only if the following conditions are satisfied:

- (boundary condition) $a * 1=a$.

- (commutativity) $a * b=b * a$.

- (increasing property) If $b<c$, then $a * b \leq a * c$.

- (associativity) $(a * b) * c=a *(b * c)$.

According to the concept of commutativity of t-norm, it is clear to see that if the t-norm is continuous with respect to the first component (resp. second component) then the t-norm is also continuous with respect to the second component (resp. first component). More precisely, given any fixed $a \in[0,1]$, we see that if the function $f(x)=a * x$ (resp. $f(x)=x * a$ ) is continuous then the function $g(x)=x * a$ (resp. $g(x)=a * x$ ) is also continuous. Regarding the one-sided continuity, it is clear to see that if the t-norm is left-continuous with respect to the first component (resp. second component), then the t-norm is also left-continuous with respect to the second component (resp. first component). Similarly, if the t-norm is right-continuous with respect to the first component (resp. second component), then the t-norm is also right-continuous with respect to the second component (resp. first component).

Proposition 1. (Wu [12]) Suppose that the t-norm * is left-continuous at 1 with respect to the first or second component. For any $a \in(0,1)$ and any $p \in \mathbb{N}$, there exists $r \in(0,1)$ such that

$$
\overbrace{r * r * \cdots * r}^{p \text { times }}>a \text {. }
$$

Definition 1. Let $X$ be a nonempty universal set, and let $M$ be a mapping defined on $X \times X \times[0, \infty)$ into $[0,1]$. Then $(X, M)$ is called a fuzzy semi-metric space if and only if the following conditions are satisfied:

- $\quad$ for any $x, y \in X, M(x, y, t)=1$ for all $t \geq 0$ if and only if $x=y$;

- $M(x, y, 0)=0$ for all $x, y \in X$ with $x \neq y$;

We say that $M$ satisfies the symmetric condition if and only if $M(x, y, t)=M(y, x, t)$ for any $x, y \in X$ and $t \geq 0$.

In general, the fuzzy semi-metric space $(X, M)$ does not necessarily satisfy the symmetric condition. Therefore four kinds of triangle inequalities are proposed below.

Definition 2. Let $X$ be a nonempty universal set, and let $M$ be a mapping defined on $X \times X \times[0, \infty)$ into $[0,1]$. Given a t-norm $*$, four different kinds of triangle inequalities are defined below. 
- We say that $M$ satisfies the $\bowtie$-triangle inequality if and only if the following inequality is satisfied:

$$
M(x, y, t) * M(y, z, s) \leq M(x, z, t+s) \text { for all } x, y, z \in X \text { and } s, t>0 .
$$

- We say that $M$ satisfies the $\triangleright$-triangle inequality if and only if the following inequality is satisfied:

$$
M(x, y, t) * M(z, y, s) \leq M(x, z, t+s) \text { for all } x, y, z \in X \text { and } s, t>0
$$

- We say that $M$ satisfies the $\triangleleft$-triangle inequality if and only if the following inequality is satisfied:

$$
M(y, x, t) * M(y, z, s) \leq M(x, z, t+s) \text { for all } x, y, z \in X \text { and } s, t>0
$$

- We say that $M$ satisfies the $\diamond$-triangle inequality if and only if the following inequality is satisfied:

$$
M(y, x, t) * M(z, y, s) \leq M(x, z, t+s) \text { for all } x, y, z \in X \text { and } s, t>0
$$

Given a fuzzy semi-metric space $(X, M)$, when we say that the mapping $M$ satisfies some kinds of triangle inequalities, it implicitly means that the t-norm $*$ is considered in $(X, M)$.

Remark 1. It is obvious that if the mapping $M$ satisfies the symmetric condition, then the concepts of $\bowtie$-triangle inequality, $\triangleright$-triangle inequality, $\triangleleft$-triangle inequality and $\diamond$-triangle inequality are all equivalent.

In this paper, we shall only study the case of assuming that $M$ satisfies the $\bowtie$-triangle inequality. The other cases of different kinds of triangle inequalities will be the future research, since they cannot be similarly investigated as the case of $\bowtie$-triangle inequality.

Remark 2. The following interesting observations will be used in the further study. Suppose that $M$ satisfies the $\bowtie$-triangle inequality. Then

$$
M\left(a, b, t_{1}\right) * M\left(b, c, t_{2}\right) * M\left(c, d, t_{3}\right) \leq M\left(a, c, t_{1}+t_{2}\right) * M\left(c, d, t_{3}\right) \leq M\left(a, d, t_{1}+t_{2}+t_{3}\right) .
$$

On the other hand, we also have

$$
M\left(b, a, t_{1}\right) * M\left(c, b, t_{2}\right)=M\left(c, b, t_{2}\right) * M\left(b, a, t_{1}\right) \leq M\left(c, a, t_{1}+t_{2}\right),
$$

which implies

$$
M\left(b, a, t_{1}\right) * M\left(c, b, t_{2}\right) * M\left(d, c, t_{3}\right) \leq M\left(d, a, t_{1}+t_{2}+t_{3}\right) .
$$

In general, we have

$$
M\left(x_{1}, x_{2}, t_{1}\right) * M\left(x_{2}, x_{3}, t_{2}\right) * \cdots * M\left(x_{p}, x_{p+1}, t_{p}\right) \leq M\left(x_{1}, x_{p+1}, t_{1}+t_{2}+\cdots+t_{p}\right)
$$

and

$$
M\left(x_{2}, x_{1}, t_{1}\right) * M\left(x_{3}, x_{2}, t_{2}\right) * \cdots * M\left(x_{p+1}, x_{p}, t_{p+1}\right) \leq M\left(x_{p+1}, x_{1}, t_{1}+t_{2}+\cdots+t_{p}\right) .
$$

Definition 3. Let $(X, M)$ be a fuzzy semi-metric space. We say that $M$ is nondecreasing if and only if, given any fixed $x, y \in X, M\left(x, y, t_{1}\right) \geq M\left(x, y, t_{2}\right)$ for $t_{1}>t_{2}$.

Proposition 2. Let $(X, M)$ be a fuzzy semi-metric space. If $M$ satisfies the $\bowtie$-triangle inequality, then $M$ is nondecreasing. 
Let $(X, d)$ be a metric space. If the sequence $\left\{x_{n}\right\}_{n=1}^{\infty}$ in $(X, d)$ converges to $x$, i.e., $d\left(x_{n}, x\right) \rightarrow 0$ as $n \rightarrow \infty$, then it is denoted by $x_{n} \stackrel{d}{\longrightarrow} x$ as $n \rightarrow \infty$. In this case, we also say that $x$ is a $d$-limit of the sequence $\left\{x_{n}\right\}_{n=1}^{\infty}$. Since $M$ does not necessarily satisfy the symmetric condition, it means that $M\left(x_{n}, x, t\right) \neq M\left(x, x_{n}, t\right)$ in general. Therefore we propose the following limit concepts.

Definition 4. Let $(X, M)$ be a fuzzy semi-metric space, and let $\left\{x_{n}\right\}_{n=1}^{\infty}$ be a sequence in $X$.

- We write $x_{n} \stackrel{M^{\triangleright}}{\longrightarrow} x$ as $n \rightarrow \infty$ if and only if

$$
\lim _{n \rightarrow \infty} M\left(x_{n}, x, t\right)=1 \text { for all } t>0 .
$$

In this case, we call $x$ a $M^{\triangleright}$-limit of the sequence $\left\{x_{n}\right\}_{n=1}^{\infty}$.

- We write $x_{n} \stackrel{M^{\triangleleft}}{\longrightarrow} x$ as $n \rightarrow \infty$ if and only if

$$
\lim _{n \rightarrow \infty} M\left(x, x_{n}, t\right)=1 \text { for all } t>0 .
$$

In this case, we call $x$ a $M^{\triangleleft}$-limit of the sequence $\left\{x_{n}\right\}_{n=1}^{\infty}$.

- We write $x_{n} \stackrel{M}{\longrightarrow} x$ as $n \rightarrow \infty$ if and only if

$$
\lim _{n \rightarrow \infty} M\left(x_{n}, x, t\right)=\lim _{n \rightarrow \infty} M\left(x, x_{n}, t\right)=1 \text { for all } t>0 .
$$

In this case, we call $x$ a $M$-limit of the sequence $\left\{x_{n}\right\}_{n=1}^{\infty}$.

The following interesting results will be used for the further study.

Proposition 3. Let $(X, M)$ be a fuzzy semi-metric space along with a $t$-norm, and let $\left\{x_{n}\right\}_{n=1}^{\infty}$ be a sequence in X. Suppose that the t-norm * is left-continuous at 1 with respect to the first or second component, and that $M$ satisfies the $\bowtie$-triangle inequality. Then we have the following properties.

- If $x_{n} \stackrel{M^{\triangleleft}}{\longrightarrow} x$ and $x_{n} \stackrel{M^{\triangleright}}{\longrightarrow} y$, then $x=y$.

- If $x_{n} \stackrel{M^{\triangleright}}{\longrightarrow} x$ and $x_{n} \stackrel{M^{\triangleleft}}{\longrightarrow} y$, then $x=y$.

Proposition 4. Let $(X, M)$ be a fuzzy semi-metric space along with a t-norm $*$ such that the $t$-norm $*$ is left-continuous with respect to the first or second component, and that satisfies the following inequality

$$
\sup _{n}\left(a_{n} * b_{n}\right) \geq\left(\sup _{n} a_{n}\right) *\left(\sup _{n} b_{n}\right)
$$

for any sequences $\left\{a_{n}\right\}_{n=1}^{\infty}$ and $\left\{b_{n}\right\}_{n=1}^{\infty}$ in $[0,1]$. Suppose that $M$ satisfies the $\bowtie$-triangle inequality. Given a sequence $\left\{\left(x_{n}, y_{n}, t_{n}\right)\right\}_{n=1}^{\infty}$ in $X \times X \times(0, \infty)$ with $t_{n} \rightarrow t^{\circ}, x_{n} \stackrel{M}{\longrightarrow} x^{\circ}$ and $y_{n} \stackrel{M}{\longrightarrow} y^{\circ}$ as $n \rightarrow \infty$, the following statements hold true.

- If the mapping $M\left(x^{\circ}, y^{\circ}, \cdot\right):(0, \infty) \rightarrow[0,1]$ is continuous at $t^{\circ}$, then

$$
\lim _{n \rightarrow \infty} M\left(x_{n}, y_{n}, t_{n}\right)=M\left(x^{\circ}, y^{\circ}, t^{\circ}\right) .
$$

- If the mapping $M\left(y^{\circ}, x^{\circ}, \cdot\right):(0, \infty) \rightarrow[0,1]$ is continuous at $t^{\circ}$, then

$$
\lim _{n \rightarrow \infty} M\left(y_{n}, x_{n}, t_{n}\right)=M\left(y^{\circ}, x^{\circ}, t^{\circ}\right) .
$$

Based on the intuitive concept of the value $M(x, y, t)$, we see that $M(x, y, t)=1$ means the distance between $x$ and $y$ that is surely less than or equal to $t$, and $M(x, y, t)=0$ means the distance between $x$ 
and $y$ that is surely greater than $t$. Therefore if $x \neq y$ with distance $t_{x y} \neq 0$ between $x$ and $y$, then it is impossible for $M(x, y, t)=0$ and for all $t>0$. In other words, there exists $t_{0}>0$ with $t_{x y}<t_{0}$ satisfying $M\left(x, y, t_{0}\right) \neq 0$. We propose the following definition.

Definition 5. Let $(X, M)$ be a fuzzy semi-metric space. We say that $M$ satisfies the distant condition if and only if, for any $x, y \in X$ with $x \neq y$, there exists $t_{0}>0$ such that $M\left(x, y, t_{0}\right) \neq 0$.

\section{Auxiliary Functions}

Since $M(x, y, t)$ is the membership degree of the distance between $x$ and $y$ that is less than or equal to $t$, it is natural to see that the mapping $M(x, y, \cdot)$ is nondecreasing or symmetrically nondecreasing as shown in Proposition 2. On the other hand, since the distance will always be less than or equal to a large $t$, it is also reasonable to argue that if $t$ is sufficiently large, then the membership degree $M(x, y, t)$ is close to 1 , and if $t$ is sufficiently small, then the membership degree $M(x, y, t)$ is close to 0 . Therefore we propose the following definition.

Definition 6. Let $(X, M)$ be a fuzzy semi-metric space.

- We say that $M$ satisfies the canonical condition if and only if, for any fixed $x, y \in X$,

$$
\lim _{t \rightarrow+\infty} M(x, y, t)=1 \text { for any fixed } x, y \in X
$$

- We say that $M$ satisfies the rational condition if and only if, for any fixed $x, y \in X$,

$$
\lim _{t \rightarrow 0+} M(x, y, t)=0 \text { for any fixed } x, y \in X
$$

Example 1. Let $X=\mathbb{R}$, and let the t-norm $*$ be taken as the multiplication $a * b=a \cdot b$. For each $t>0$, we define

$$
M(x, y, t)=\frac{t}{t+|x-y|}
$$

for all $x, y \in \mathbb{R}$. Then $(X, M, *)$ is a fuzzy metric space such that $M$ satisfies the canonical and rational conditions, and that the mapping $M(x, y, \cdot):(0,+\infty) \rightarrow[0,1]$ is continuous.

Given a sequence $\left\{x_{n}\right\}_{n=1}^{\infty}$ in fuzzy semi-metric space $(X, M)$, we see that $M\left(x_{m}, x_{n}, t\right) \neq$ $M\left(x_{n}, x_{m}, t\right)$ in general. Therefore the different concepts of Cauchy sequence are proposed below.

Definition 7. Let $(X, M)$ be a fuzzy semi-metric space, and let $\left\{x_{n}\right\}_{n=1}^{\infty}$ be a sequence in $X$.

- We say that $\left\{x_{n}\right\}_{n=1}^{\infty}$ is a $>$-Cauchy sequence if and only if, given any pair $(r, t)$ with $t>0$ and $0<r<1$, there exists $n_{r, t} \in \mathbb{N}$ such that $M\left(x_{m}, x_{n}, t\right)>1-r$ for all pairs $(m, n)$ of integers $m$ and $n$ with $m>n \geq n_{r, t}$.

- We say that $\left\{x_{n}\right\}_{n=1}^{\infty}$ is a $<$-Cauchy sequence if and only if, given any pair $(r, t)$ with $t>0$ and $0<r<1$, there exists $n_{r, t} \in \mathbb{N}$ such that $M\left(x_{n}, x_{m}, t\right)>1-r$ for all pairs $(m, n)$ of integers $m$ and $n$ with $m>n \geq n_{r, t}$.

The $>$-Cauchy sequence means that we consider $M\left(x_{m}, x_{n}, t\right)>1-r$ for $m>n$, and the $<-$ Cauchy sequence means that we consider $M\left(x_{n}, x_{m}, t\right)>1-r$ for $n<m$.

- We say that $\left\{x_{n}\right\}_{n=1}^{\infty}$ is a Cauchy sequence if and only if, given any pair $(r, t)$ with $t>0$ and $0<r<1$, there exists $n_{r, t} \in \mathbb{N}$ such that $M\left(x_{m}, x_{n}, t\right)>1-r$ and $M\left(x_{n}, x_{m}, t\right)>1-r$ for all pairs $(m, n)$ of integers $m$ and $n$ with $m, n \geq n_{r, t}$ and $m \neq n$.

The theorems of coincidence points and fixed points should be based on the Cauchy sequences. Therefore we introduce two kinds of auxiliary functions to obtain the useful properties regarding the 
Cauchy sequences. One is based on the infimum, and another one is based on the supremum. If $M$ satisfies the canonical condition, then we shall consider the auxiliary function using the concept of infimum, and if $M$ satisfies the rational condition, then we shall consider the auxiliary function using the concept of supremum.

\subsection{Auxiliary Function Based on the Infimum}

Let $(X, M)$ be a fuzzy semi-metric space such that $M$ satisfies the canonical condition. We introduce the auxiliary function using the concept of infimum.

Definition 8. Let $(X, M)$ be a fuzzy semi-metric space such that $M$ satisfies the canonical condition. Given any fixed $x, y \in X$ and any fixed $\lambda \in(0,1]$, we define the set

$$
\Omega^{\downarrow}(\lambda, x, y)=\{t>0: M(x, y, t) \geq 1-\lambda\}
$$

and the function $\Gamma^{\downarrow}(\lambda, \cdot, \cdot): X \times X \rightarrow[0,+\infty)$ by

$$
\Gamma^{\downarrow}(\lambda, x, y)=\inf \Omega^{\downarrow}(\lambda, x, y)=\inf \{t>0: M(x, y, t) \geq 1-\lambda\} .
$$

Since the symmetric condition is not satisfied, it means that $\Omega^{\downarrow}(\lambda, x, y) \neq \Omega^{\downarrow}(\lambda, y, x)$ and $\Gamma^{\downarrow}(\lambda, x, y) \neq \Gamma^{\downarrow}(\lambda, y, x)$ in general. We also need to claim that $\Omega^{\downarrow}(\lambda, x, y) \neq \varnothing$ and $\Omega^{\downarrow}(\lambda, y, x) \neq \varnothing$. Suppose that $\Omega^{\downarrow}(\lambda, x, y)=\varnothing$. Then we must have $M(x, y, t)<1-\lambda$ for all $t>0$. Therefore we obtain

$$
\lim _{t \rightarrow+\infty} M(x, y, t) \leq 1-\lambda<1,
$$

which contradicts the fact that $M$ satisfies the canonical condition. This says that Definition 8 is well-defined.

Proposition 5. Let $(X, M)$ be a fuzzy semi-metric space such that $M$ satisfies the canonical condition. Suppose that $M$ satisfies the $\bowtie$-triangle inequality. Given any fixed $x, y \in X$ and any fixed $\lambda \in(0,1)$, for any $\epsilon>0$, we have

$$
M\left(x, y, \Gamma^{\downarrow}(\lambda, x, y)+\epsilon\right) \geq 1-\lambda .
$$

If we further assume that the mapping $M(x, y, \cdot):(0, \infty) \rightarrow[0,1]$ is right-continuous, then

$$
M\left(x, y, \Gamma^{\downarrow}(\lambda, x, y)\right) \geq 1-\lambda .
$$

Proposition 6. Let $(X, M)$ be a fuzzy semi-metric space along with a t-norm $*$ such that $M$ satisfies the canonical condition and the $t$-norm $*$ is left-continuous at 1 in the first or second component. Suppose that $M$ satisfies the $\bowtie$-triangle inequality. Given any fixed $x_{1}, x_{2}, \cdots, x_{p} \in X$ and any fixed $\mu \in(0,1]$, there exists $\lambda \in(0,1)$ such that

$$
\Gamma^{\downarrow}\left(\mu, x_{1}, x_{p}\right) \leq \Gamma^{\downarrow}\left(\lambda, x_{1}, x_{2}\right)+\Gamma^{\downarrow}\left(\lambda, x_{2}, x_{3}\right)+\Gamma^{\downarrow}\left(\lambda, x_{3}, x_{4}\right)+\cdots+\Gamma^{\downarrow}\left(\lambda, x_{p-1}, x_{p}\right)
$$

and

$$
\Gamma^{\downarrow}\left(\mu, x_{p}, x_{1}\right) \leq \Gamma^{\downarrow}\left(\lambda, x_{2}, x_{1}\right)+\Gamma^{\downarrow}\left(\lambda, x_{3}, x_{2}\right)+\Gamma^{\downarrow}\left(\lambda, x_{4}, x_{3}\right)+\cdots+\Gamma^{\downarrow}\left(\lambda, x_{p}, x_{p-1}\right) .
$$

Proof. If $\mu=1$, then

$$
\Gamma^{\downarrow}\left(1, x_{1}, x_{p}\right)=\inf \left\{t>0: M\left(x_{1}, x_{p}, t\right) \geq 0\right\}=\inf \{t>0\}=0,
$$


and the result is obvious. Therefore we assume $\mu \in(0,1)$. According to Proposition 1 , there exists $\lambda \in(0,1)$ such that

$$
(1-\lambda) * \cdots *(1-\lambda)>1-\mu
$$

Given any $\epsilon>0$, we have

$$
\begin{aligned}
& M\left(x_{1}, x_{p}, \Gamma^{\downarrow}\left(\lambda, x_{1}, x_{2}\right)+\Gamma^{\downarrow}\left(\lambda, x_{2}, x_{3}\right)+\cdots+\Gamma^{\downarrow}\left(\lambda, x_{p-1}, x_{p}\right)+(p-1) \epsilon\right) \\
& \quad \geq M\left(x_{1}, x_{2}, \Gamma^{\downarrow}\left(\lambda, x_{1}, x_{2}\right)+\epsilon\right) * \cdots * M\left(x_{p-1}, x_{p}, \Gamma^{\downarrow}\left(\lambda, x_{p-1}, x_{p}\right)+\epsilon\right)
\end{aligned}
$$

(by Remark 2)

$\geq(1-\lambda) * \cdots *(1-\lambda)$ (by $(1)$ and the increasing property of $\mathrm{t}$-norm)

$>1-\mu($ by $(5))$.

By the definition of $\Gamma_{\lambda}$, we obtain

$$
\Gamma^{\downarrow}\left(\lambda, x_{1}, x_{2}\right)+\Gamma^{\downarrow}\left(\lambda, x_{2}, x_{3}\right)+\cdots+\Gamma^{\downarrow}\left(\lambda, x_{p-1}, x_{p}\right)+(p-1) \epsilon \geq \Gamma^{\downarrow}\left(\mu, x_{1}, x_{p}\right) .
$$

By taking $\epsilon \rightarrow 0+$, we obtain the inequality (3). The inequality (4) can be similarly obtained. This completes the proof.

Definition 9. Let $(X, M)$ be a fuzzy semi-metric space such that $M$ satisfies the canonical condition.

- $\quad$ Given any fixed $\lambda \in(0,1)$, we say that $\left\{x_{n}\right\}_{n=1}^{\infty}$ is a $(\lambda,>)$-Cauchy sequence with respect to $\Gamma^{\downarrow}$ if and only if, given any $\epsilon>0$, there exists $n_{\epsilon} \in \mathbb{N}$ such that $m>n \geq n_{\epsilon}$ implies $\Gamma^{\downarrow}\left(\lambda, x_{m}, x_{n}\right)<\epsilon$.

- $\quad$ Given any fixed $\lambda \in(0,1)$, we say that $\left\{x_{n}\right\}_{n=1}^{\infty}$ is a $(\lambda,<)$-Cauchy sequence with respect to $\Gamma^{\downarrow}$ if and only if, given any $\epsilon>0$, there exists $n_{\epsilon} \in \mathbb{N}$ such that $n>m \geq n_{\epsilon}$ implies $\Gamma^{\downarrow}\left(\lambda, x_{m}, x_{n}\right)<\epsilon$.

- Given any fixed $\lambda \in(0,1)$, we say that $\left\{x_{n}\right\}_{n=1}^{\infty}$ is a $\lambda$-Cauchy sequence with respect to $\Gamma^{\downarrow}$ if and only if, given any $\epsilon>0$, there exists $n_{\epsilon} \in \mathbb{N}$ such that $m, n \geq n_{\epsilon}$ implies $\Gamma^{\downarrow}\left(\lambda, x_{m}, x_{n}\right)<\epsilon$ and $\Gamma^{\downarrow}\left(\lambda, x_{n}, x_{m}\right)<\epsilon$.

The following interesting properties will be used for the further study.

Proposition 7. Let $(X, M)$ be a fuzzy semi-metric space along with a t-norm $*$ such that $M$ satisfies the canonical condition and the t-norm $*$ is left-continuous at 1 with respect to the first or second component. Suppose that $M$ satisfies the $\bowtie$-triangle inequality.

(i) Let $\left\{x_{n}\right\}_{n=1}^{\infty}$ be a sequence in $X$. Then the following statements hold true.

- $\quad x_{n} \stackrel{M^{\triangleright}}{\longrightarrow} x$ as $n \rightarrow \infty$ if and only if $\Gamma^{\downarrow}\left(\lambda, x_{n}, x\right) \rightarrow 0$ as $n \rightarrow \infty$ for all $\lambda \in(0,1)$.

- $\quad x_{n} \stackrel{M^{\triangleleft}}{\longrightarrow} x$ as $n \rightarrow \infty$ if and only if $\Gamma^{\downarrow}\left(\lambda, x, x_{n}\right) \rightarrow 0$ as $n \rightarrow \infty$ for all $\lambda \in(0,1)$.

(ii) Let $\left\{x_{n}\right\}_{n=1}^{\infty}$ be a sequence in $X$. Then the following statements hold true.

- $\left\{x_{n}\right\}_{n=1}^{\infty}$ is a >-Cauchy sequence in metric sense if and only if it is a $(\lambda,>)$-Cauchy sequence with respect to $\Gamma^{\downarrow}$ for all $\lambda \in(0,1)$.

- $\left\{x_{n}\right\}_{n=1}^{\infty}$ is a -Cauchy sequence in metric sense if and only if it is a $(\lambda,<)$-Cauchy sequence with respect to $\Gamma^{\downarrow}$ for all $\lambda \in(0,1)$.

\subsection{Auxiliary Function Based on the Supremum}

Let $(X, M)$ be a fuzzy semi-metric space such that $M$ satisfies the rational condition. We introduce the auxiliary function using the concept of supremum. 
Definition 10. Let $(X, M)$ be a fuzzy semi-metric space such that $M$ satisfies the rational condition. Given any fixed $x, y \in X$ with $x \neq y$ and any fixed $\lambda \in[0,1)$, we define the set

$$
\Omega^{\uparrow}(\lambda, x, y)=\{t>0: M(x, y, t) \leq 1-\lambda\}
$$

and the function $\Gamma^{\uparrow}(\lambda, \cdot, \cdot): X \times X \rightarrow[0,+\infty)$ by

$$
\Gamma^{\uparrow}(\lambda, x, y)=\sup \Omega^{\uparrow}(\lambda, x, y)=\sup \{t>0: M(x, y, t) \leq 1-\lambda\}
$$

For convenience, we also define $\Gamma^{\uparrow}(\lambda, x, x)=0$ for all $\lambda \in[0,1)$.

For $x \neq y$, we need to claim that $\Omega^{\uparrow}(\lambda, x, y) \neq \varnothing$. Suppose that $\Omega^{\uparrow}(\lambda, x, y)=\varnothing$. Then we must have $M(x, y, t)>1-\lambda$ for all $t>0$. Therefore we obtain

$$
\lim _{t \rightarrow 0+} M(x, y, t) \geq 1-\lambda>0
$$

which contradicts the fact that $M$ satisfies the the rational condition. This says that Definition 10 is well-defined. We also see that

$$
\Gamma^{\uparrow}(0, x, y)=\sup \{t>0: M(x, y, t) \leq 1\}=\sup \{t>0\}=+\infty
$$

Let us recall that $M(x, y, t)$ can be interpreted as the membership degree of the distance between $x$ and $y$ that is less than or equal to $t$. It means that, when the distance between $x$ and $y$ is finite and $t_{x y}$ is a positive real number that is larger than the distance between $x$ and $y$, we should have $M\left(x, y, t_{x y}\right)=1$. In other words, the distance between $x$ and $y$ that is less than or equal to $t_{x y}$ is definitely true. The formal definition is given below.

Definition 11. (Wu [12]) Let $(X, M, *)$ be a fuzzy quasi-metric space. Given any two elements $x, y \in X$ with $x \neq y$, the concepts of finite distance are defined below.

- We say that $x$ and $y$ have a finite distance beginning from $x$ to $y$ if and only if there exists $t_{x y} \in(0,+\infty)$ such that $M\left(x, y, t_{x y}\right)=1$.

- We say that $x$ and $y$ have a finite distance if and only if $x$ and $y$ have a finite distance beginning from $x$ to $y$ and beginning from $y$ to $x$.

It is obvious that if $M$ satisfies the symmetric condition, then the concepts of finite distance beginning from $x$ to $y$ and beginning from $y$ to $x$ are equivalent. The following results can be obtained by using the routine argument.

Proposition 8. Let $(X, M)$ be a fuzzy semi-metric space along with a t-norm $*$ such that $M$ satisfies the rational condition. Given any fixed $x, y \in X$ and any fixed $\lambda \in(0,1)$ such that $\Gamma^{\uparrow}(\lambda, x, y)<+\infty$, for any $\epsilon>0$, we have

$$
M\left(x, y, \Gamma^{\uparrow}(\lambda, x, y)+\epsilon\right)>1-\lambda
$$

Proposition 9. Let $(X, M)$ be a fuzzy semi-metric space along with a t-norm $*$ such that $M$ satisfies the rational condition. Suppose that $M$ satisfies the $\bowtie$-triangle inequality. Given any fixed $x, y \in X$ and any fixed $\lambda \in(0,1)$, we have the following properties.

(i) If $M(x, y, t) \leq 1-\lambda$ then $t \leq \Gamma^{\uparrow}(\lambda, x, y)$.

(ii) If $M(x, y, t)>1-\lambda$, then $\Gamma^{\uparrow}(\lambda, x, y)<+\infty$ and $t \geq \Gamma^{\uparrow}(\lambda, x, y)$.

Proposition 10. Let $(X, M)$ be a fuzzy semi-metric space along with a $t$-norm $*$ such that the $t$-norm is left-continuous at 1 in the first or second component. Suppose that $M$ satisfies the rational condition and the 
$\bowtie$-triangle inequality. Given any fixed $x_{1}, x_{2}, \cdots, x_{p} \in X$ and any fixed $\mu \in(0,1)$, there exists $\lambda \in(0,1)$ such that

$$
\Gamma^{\uparrow}\left(\mu, x_{1}, x_{p}\right) \leq \Gamma^{\uparrow}\left(\lambda, x_{1}, x_{2}\right)+\Gamma^{\uparrow}\left(\lambda, x_{2}, x_{3}\right)+\cdots+\Gamma^{\uparrow}\left(\lambda, x_{p-1}, x_{p}\right) .
$$

and

$$
\Gamma^{\uparrow}\left(\mu, x_{p}, x_{1}\right) \leq \Gamma^{\uparrow}\left(\lambda, x_{2}, x_{1}\right)+\Gamma^{\uparrow}\left(\lambda, x_{3}, x_{2}\right)+\cdots+\Gamma^{\uparrow}\left(\lambda, x_{p}, x_{p-1}\right) .
$$

Proof. Using Proposition 1 , it is clear to see that there exists $\lambda \in(0,1)$ such that

$$
(1-\lambda) * \cdots *(1-\lambda)>1-\mu
$$

Suppose that $\Gamma^{\uparrow}\left(\lambda, x_{i}, x_{i+1}\right)<+\infty$ for all $i=1, \cdots, p-1$. Given any $\epsilon>0$, we have

$$
\begin{aligned}
M & \left(x_{1}, x_{p}, \Gamma^{\uparrow}\left(\lambda, x_{1}, x_{2}\right)+\Gamma^{\uparrow}\left(\lambda, x_{2}, x_{3}\right)+\cdots+\Gamma^{\uparrow}\left(\lambda, x_{p-1}, x_{p}\right)+(p-1) \epsilon\right) \\
\geq & M\left(x_{1}, x_{2}, \Gamma^{\uparrow}\left(\lambda, x_{1}, x_{2}\right)+\epsilon\right) * \cdots * M\left(x_{p-1}, x_{p}, \Gamma^{\uparrow}\left(\lambda, x_{p-1}, x_{p}\right)+\epsilon\right) \\
& (\text { by Remark 2) } \\
\geq & (1-\lambda) * \cdots *(1-\lambda) \text { (by (6) and the increasing property of t-norm) } \\
> & 1-\mu \text { (by (9)). }
\end{aligned}
$$

We consider the following cases.

- Assume that $\Gamma^{\uparrow}\left(\mu, x_{1}, x_{p}\right)=+\infty$. We need to claim that there exists $i_{0}$ satisfying $\Gamma^{\uparrow}\left(\lambda, x_{i_{0}}, x_{i_{0}+1}\right)=+\infty$. Suppose that $\Gamma^{\uparrow}\left(\lambda, x_{i}, x_{i+1}\right)<+\infty$ for all $i=1, \cdots, p-1$. From (10) and part (i) of Proposition 9, we have $\Gamma^{\uparrow}\left(\mu, x_{1}, x_{p}\right)<+\infty$, which contradicts the fact that there exists $i_{0}$ satisfying $\Gamma^{\uparrow}\left(\lambda, x_{i_{0}}, x_{i_{0}+1}\right)=+\infty$. This shows that the inequality (7) holds true.

- Assume that $\Gamma^{\uparrow}\left(\mu, x_{1}, x_{p}\right)<+\infty$. The following cases will also be considered.

- $\quad$ Suppose that there exists $i_{0}$ satisfying $\Gamma^{\uparrow}\left(\lambda, x_{i_{0}}, x_{i_{0}+1}\right)=+\infty$. Then the inequality (7) also holds true.

- $\quad$ Suppose that $\Gamma^{\uparrow}\left(\lambda, x_{i}, x_{i+1}\right)<+\infty$ for all $i=1, \cdots, p-1$. Then, using (10) and part (ii) of Proposition 9 again, we can obtain

$$
\Gamma^{\uparrow}\left(\lambda, x_{1}, x_{2}\right)+\Gamma^{\uparrow}\left(\lambda, x_{2}, x_{3}\right)+\cdots+\Gamma^{\uparrow}\left(\lambda, x_{p-1}, x_{p}\right)+(p-1) \epsilon \geq \Gamma^{\uparrow}\left(\mu, x_{1}, x_{p}\right) .
$$

By taking $\epsilon \rightarrow 0+$, it follows that the desired inequality (7) can be obtained.

Using Remark 2, we can similarly obtain the inequality (8). This completes the proof.

Definition 12. Let $(X, M)$ be a fuzzy semi-metric space along with a t-norm $*$ such that $M$ satisfies the rational condition.

- Given any fixed $\lambda \in(0,1)$, we say that $\left\{x_{n}\right\}_{n=1}^{\infty}$ is a $(\lambda,>)$-Cauchy sequence with respect to $\Gamma^{\uparrow}$ if and only if, given any $\epsilon>0$, there exists $n_{\epsilon} \in \mathbb{N}$ such that $m>n \geq n_{\epsilon}$ implies $\Gamma^{\uparrow}\left(\lambda, x_{m}, x_{n}\right)<\epsilon$.

- Given any fixed $\lambda \in(0,1)$, we say that $\left\{x_{n}\right\}_{n=1}^{\infty}$ is a $(\lambda,<)$-Cauchy sequence with respect to $\Gamma^{\uparrow}$ if and only if, given any $\epsilon>0$, there exists $n_{\epsilon} \in \mathbb{N}$ such that $n>m \geq n_{\epsilon}$ implies $\Gamma^{\uparrow}\left(\lambda, x_{m}, x_{n}\right)<\epsilon$.

- Given any fixed $\lambda \in(0,1)$, we say that $\left\{x_{n}\right\}_{n=1}^{\infty}$ is a $\lambda$-Cauchy sequence with respect to $\Gamma^{\uparrow}$ if and only if, given any $\epsilon>0$, there exists $n_{\epsilon} \in \mathbb{N}$ such that $m, n \geq n_{\epsilon}$ implies $\Gamma^{\uparrow}\left(\lambda, x_{m}, x_{n}\right)<\epsilon$.

The following interesting properties that can be obtained by using the routine argument will be used for the further study. 
Proposition 11. Let $(X, M)$ be a fuzzy semi-metric space along with a t-norm $*$ such that the $t$-norm $*$ is left-continuous at 1 with respect to the first or second component. Suppose that $M$ satisfies the rational condition and the $\bowtie$-triangle inequality. Let $\left\{x_{n}\right\}_{n=1}^{\infty}$ be a sequence in $X$. Then we have the following results.

(i) $\left\{x_{n}\right\}_{n=1}^{\infty}$ is a >-Cauchy sequence in metric sense if and only if it is a $(\lambda,>)$-Cauchy sequence with respect to $\Gamma^{\uparrow}$ for all $\lambda \in(0,1)$.

(ii) $\left\{x_{n}\right\}_{n=1}^{\infty}$ is a $<$-Cauchy sequence in metric sense if and only if it is a $(\lambda,<)$-Cauchy sequence with respect to $\Gamma^{\uparrow}$ for all $\lambda \in(0,1)$.

\section{Cauchy Sequences}

We shall present many kinds of situations that can guarantee the Cauchy sequence in order to derive the theorems of common coincidence points and common fixed points. We first consider the auxiliary function $\Gamma^{\downarrow}$ by assuming that $M$ satisfies the canonical condition.

Proposition 12. Let $(X, M)$ be a fuzzy semi-metric space along with a $t$-norm $*$ such that the $t$-norm $*$ is left-continuous at 1 with respect to the first or second component. Suppose that $M$ satisfies the canonical condition and the $\bowtie$-triangle inequality. Let $0<k<1$ be any fixed constant, and let $\left\{x_{n}\right\}_{n=1}^{\infty}$ be a sequence in $X$. Then we have the following results.

(i) Assume that there exist fixed elements $a_{1}, b_{1} \in X$ such that

$$
\sup _{\lambda \in(0,1)} \Gamma^{\downarrow}\left(\lambda, a_{1}, b_{1}\right)<+\infty \text { and } M\left(x_{n}, x_{n+1}, t\right) \geq M\left(a_{1}, b_{1}, \frac{t}{k^{n}}\right) \text { for all } n \in \mathbb{N} \text {. }
$$

Then $\left\{x_{n}\right\}_{n=1}^{\infty}$ is a <-Cauchy sequence.

(ii) Assume that there exist fixed elements $a_{2}, b_{2} \in X$ such that

$$
\sup _{\lambda \in(0,1)} \Gamma^{\downarrow}\left(\lambda, a_{2}, b_{2}\right)<+\infty \text { and } M\left(x_{n+1}, x_{n}, t\right) \geq M\left(a_{2}, b_{2}, \frac{t}{k^{n}}\right) \text { for all } n \in \mathbb{N} \text {. }
$$

Then $\left\{x_{n}\right\}_{n=1}^{\infty}$ is a >-Cauchy sequence.

Proof. To prove part (i) of this proposition, given any $\lambda \in(0,1)$, we have the following inclusion

$$
\left\{t>0: M\left(a_{1}, b_{1}, \frac{t}{k^{n}}\right) \geq 1-\lambda\right\} \subseteq\left\{t>0: M\left(x_{n}, x_{n+1}, t\right) \geq 1-\lambda\right\} .
$$

Therefore we obtain

$$
\begin{aligned}
\Gamma^{\downarrow}\left(\lambda, x_{n}, x_{n+1}\right) & =\inf \left\{t>0: M\left(x_{n}, x_{n+1}, t\right) \geq 1-\lambda\right\} \\
& \leq \inf \left\{t>0: M\left(a_{1}, b_{1}, \frac{t}{k^{n}}\right) \geq 1-\lambda\right\} \text { (by (13)) } \\
& =\inf \left\{k^{n} \cdot t>0: M\left(a_{1}, b_{1}, t\right) \geq 1-\lambda\right\} \\
& =k^{n} \cdot \inf \left\{t>0: M\left(a_{1}, b_{1}, t\right) \geq 1-\lambda\right\} \\
& =k^{n} \cdot \Gamma^{\downarrow}\left(\lambda, a_{1}, b_{1}\right) .
\end{aligned}
$$

Now we assume that $m, n \in \mathbb{N}$ with $m>n$. Given any $\mu \in(0,1)$, by Proposition 6 , there exists $\lambda \in(0,1)$ (which depends on $m$ and $n$ ) such that 


$$
\begin{aligned}
\Gamma^{\downarrow}\left(\mu, x_{n}, x_{m}\right) & \leq \Gamma^{\downarrow}\left(\lambda, x_{n}, x_{n+1}\right)+\Gamma^{\downarrow}\left(\lambda, x_{n+1}, x_{n+2}\right)+\cdots+\Gamma^{\downarrow}\left(\lambda, x_{m-1}, x_{m}\right) \\
& \leq k^{n} \cdot \Gamma^{\downarrow}\left(\lambda, a_{1}, b_{1}\right)+k^{n+1} \cdot \Gamma^{\downarrow}\left(\lambda, a_{1}, b_{1}\right)+\cdots+k^{m-1} \cdot \Gamma^{\downarrow}\left(\lambda, a_{1}, b_{1}\right) \text { (by (14)) } \\
& =\Gamma^{\downarrow}\left(\lambda, a_{1}, b_{1}\right) \cdot \frac{k^{n} \cdot\left(1-k^{m-n}\right)}{1-k} \leq \Gamma^{\downarrow}\left(\lambda, a_{1}, b_{1}\right) \cdot \frac{k^{n}}{1-k} \\
& \leq\left[\sup _{\lambda \in(0,1)} \Gamma^{\downarrow}\left(\lambda, a_{1}, b_{1}\right)\right] \cdot \frac{k^{n}}{1-k} \rightarrow 0 \text { as } n \rightarrow \infty,
\end{aligned}
$$

which shows that $\left\{x_{n}\right\}_{n=1}^{\infty}$ is a $(\mu,<)$-Cauchy sequence with respect to $\Gamma^{\downarrow}$ for all $\mu \in(0,1)$. By part (ii) of Proposition 7, we obtain the desired result. For proving part (ii) of this proposition, we consider

$$
\Gamma^{\downarrow}\left(\mu, x_{m}, x_{n}\right) \leq \Gamma^{\downarrow}\left(\lambda, x_{m}, x_{m-1}\right)+\Gamma^{\downarrow}\left(\lambda, x_{m-1}, x_{m-2}\right)+\cdots+\Gamma^{\downarrow}\left(\lambda, x_{n+1}, x_{n}\right) .
$$

Then we can similarly obtain the desired result by using the above argument. This completes the proof.

Next we consider the auxiliary function $\Gamma^{\uparrow}$ by assuming that $M$ satisfies the rational condition.

Proposition 13. Let $(X, M)$ be a fuzzy semi-metric space along with a $t$-norm $*$ such that the $t$-norm $*$ is left-continuous at 1 with respect to the first or second component. Suppose that $M$ satisfies the rational condition and $\bowtie$-triangle inequality. Let $0<k<1$ be any fixed constant, and let $\left\{x_{n}\right\}_{n=1}^{\infty}$ be a sequence in $X$.

(i) Assume that there exist fixed elements $a_{1}, b_{1} \in X$ such that

$$
M\left(x_{n}, x_{n+1}, t\right) \geq M\left(a_{1}, b_{1}, \frac{t}{k^{n}}\right) \text { for all } n \in \mathbb{N},
$$

and that, given any $\lambda \in(0,1)$, there exists $t_{\lambda}>0$ such that

$$
M\left(a_{1}, b_{1}, t_{\lambda}\right)>1-\lambda \text { and } \sup _{\lambda \in(0,1)} t_{\lambda}<+\infty .
$$

Then $\left\{x_{n}\right\}_{n=1}^{\infty}$ is a $<$-Cauchy sequence.

(ii) Assume that there exist fixed elements $a_{2}, b_{2} \in X$ such that

$$
M\left(x_{n+1}, x_{n}, t\right) \geq M\left(a_{2}, b_{2}, \frac{t}{k^{n}}\right) \text { for all } n \in \mathbb{N},
$$

and that, given any $\lambda \in(0,1)$, there exists $t_{\lambda}>0$ such that

$$
M\left(a_{2}, b_{2}, t_{\lambda}\right)>1-\lambda \text { and } \sup _{\lambda \in(0,1)} t_{\lambda}<+\infty .
$$

Then $\left\{x_{n}\right\}_{n=1}^{\infty}$ is a >-Cauchy sequence.

Proof. We just prove part (i) of this proposition. Given $n \in \mathbb{N}$, let $t_{n, \lambda}=k^{n} \cdot t_{\lambda}$. From (16), we see that

$$
M\left(x_{n}, x_{n+1}, t_{n, \lambda}\right) \geq M\left(a_{1}, b_{1}, t_{\lambda}\right)>1-\lambda .
$$

Using part (ii) of Proposition 9, the inequality (17) says that

$$
\Gamma^{\uparrow}\left(\lambda, x_{n}, x_{n+1}\right) \leq t_{n, \lambda} \text { for all } n \in \mathbb{N} .
$$


Now we consider $m, n \in \mathbb{N}$ with $m>n$. Given any $\mu \in(0,1)$, using (18) and Proposition 10, there exists $\lambda \in(0,1)$ (which depends on $m$ and $n$ ) such that

$$
\begin{aligned}
\Gamma^{\uparrow}\left(\mu, x_{n}, x_{m}\right) & \leq \Gamma^{\uparrow}\left(\lambda, x_{n}, x_{n+1}\right)+\Gamma^{\uparrow}\left(\lambda, x_{n+1}, x_{n+2}\right)+\cdots+\Gamma^{\uparrow}\left(\lambda, x_{m-1}, x_{m}\right) \\
& \leq k^{n} \cdot t_{\lambda}+k^{n+1} \cdot t_{\lambda}+\cdots+k^{m-1} \cdot t_{\lambda} \\
& =t_{\lambda} \cdot \frac{k^{n} \cdot\left(1-k^{m-n}\right)}{1-k} \leq t_{\lambda} \cdot \frac{k^{n}}{1-k} \\
& \leq\left(\sup _{\lambda \in(0,1)} t_{\lambda}\right) \cdot \frac{k^{n}}{1-k} \rightarrow 0 \text { as } n \rightarrow \infty,
\end{aligned}
$$

which shows that $\left\{x_{n}\right\}_{n=1}^{\infty}$ is a $(\mu,<)$-Cauchy sequence with respect to $\Gamma^{\uparrow}$ for all $\mu \in(0,1)$. By Proposition 11, we obtain the desired result. This completes the proof.

\section{Common Coincidence Points}

Now we are in a position to present the theorems of common coincidence points. Let $X$ be a nonempty universal set. We consider the mappings $T: X \rightarrow X$ and $f: X \rightarrow X$.

- We say that the mappings $T$ and $f$ commute if and only if $f(T(x))=T(f(x))$ for all $x, y \in X$.

- An element $x \in X$ is called a coincidence point of mappings $T$ and $f$ if and only if $T(x)=f(x)$. In particular, if $x=f(x)=T(x)$, then $x$ is called a common fixed point of $T$ and $f$.

Definition 13. Let $(X, M)$ be a fuzzy semi-metric space.

- We say that the function $f: X \rightarrow X$ is $(\triangleright, \triangleright)$-continuous with respect to $M$ if and only if, given any sequence $\left\{x_{n}\right\}_{n=1}^{\infty}$ in $X, x_{n} \stackrel{M^{\triangleright}}{\longrightarrow} x$ as $n \rightarrow \infty$ implies $f\left(x_{n}\right) \stackrel{M^{\triangleright}}{\longrightarrow} f(x)$ as $n \rightarrow \infty$.

- We say that the function $f: X \rightarrow X$ is $(\triangleright, \triangleleft)$-continuous with respect to $M$ if and only if, given any sequence $\left\{x_{n}\right\}_{n=1}^{\infty}$ in $X, x_{n} \stackrel{M^{\triangleright}}{\longrightarrow} x$ as $n \rightarrow \infty$ implies $f\left(x_{n}\right) \stackrel{M^{\triangleleft}}{\longrightarrow} f(x)$ as $n \rightarrow \infty$.

- We say that the function $f: X \rightarrow X$ is $(\triangleleft, \triangleright)$-continuous with respect to $M$ if and only if, given any sequence $\left\{x_{n}\right\}_{n=1}^{\infty}$ in $X, x_{n} \stackrel{M^{\triangleleft}}{\longrightarrow} x$ as $n \rightarrow \infty$ implies $f\left(x_{n}\right) \stackrel{M^{\triangleright}}{\longrightarrow} f(x)$ as $n \rightarrow \infty$.

- We say that the function $f: X \rightarrow X$ is $(\triangleleft, \triangleleft)$-continuous with respect to $M$ if and only if, given any sequence $\left\{x_{n}\right\}_{n=1}^{\infty}$ in $X, x_{n} \stackrel{M^{\triangleleft}}{\longrightarrow} x$ as $n \rightarrow \infty$ implies $f\left(x_{n}\right) \stackrel{M^{\triangleleft}}{\longrightarrow} f(x)$ as $n \rightarrow \infty$.

Definition 14. Let $(X, M)$ be a fuzzy semi-metric space.

- We say that $(X, M)$ is $(>, \triangleright)$-complete if and only if each $>$-Cauchy sequence is convergent in the sense of $x_{n} \stackrel{M^{\triangleright}}{\longrightarrow} x$.

- We say that $(X, M)$ is $(>, \triangleleft)$-complete if and only if each $>$-Cauchy sequence is convergent in the sense of $x_{n} \stackrel{M^{\triangleleft}}{\longrightarrow} x$.

- We say that $(X, M)$ is $(<, \triangleright)$-complete if and only if each $<$-Cauchy sequence is convergent in the sense of $x_{n} \stackrel{M^{\triangleright}}{\longrightarrow} x$.

- We say that $(X, M)$ is $(<, \triangleleft)$-complete if and only if each $<$-Cauchy sequence is convergent in the sense of $x_{n} \stackrel{M^{\triangleleft}}{\longrightarrow} x$.

Let $X$ be a nonempty universal set, and let $T: X \rightarrow X$ be a mapping from $X$ into itself. We write the composition $T^{2}=T \circ T$ and $T^{m}=T \circ T^{m-1}$ for $m \in \mathbb{N}$ and $m \geq 3$. Let $\left\{T_{n}\right\}_{n=1}^{\infty}$ be a sequence of mappings defined on $X$ into itself. For some $m \in \mathbb{N}$, suppose that the mapping $f: X \rightarrow X$ satisfies $T_{n}^{m}(X) \subseteq f(X)$ for all $n \in \mathbb{N}$. Given any $x_{0} \in X$, since $T_{1}^{m}(X) \subseteq f(X)$, there exists $x_{1} \in X$ such that 
$f\left(x_{1}\right)=T_{1}^{m}\left(x_{0}\right)$. Similarly, there also exists $x_{2} \in X$ such that $f\left(x_{2}\right)=T_{2}^{m}\left(x_{1}\right)$. Continuing this process, we can construct a sequence $\left\{x_{n}\right\}_{n=1}^{\infty}$ satisfying

$$
f\left(x_{n}\right)=T_{n}^{m}\left(x_{n-1}\right)
$$

for all $n \in \mathbb{N}$.

Theorem 1. Let $(X, M)$ be a fuzzy semi-metric space along with a t-norm $*$ such that $M$ satisfies the rational condition and the $\bowtie$-triangle inequality. Suppose that the following conditions are satisfied:

- the t-norm is left-continuous at 1 in the first or second component;

- for any fixed $x, y \in X$, the mapping $M(x, y, \cdot):(0, \infty) \rightarrow[0,1]$ is left-continuous at each point $t \in(0, \infty)$;

- given any fixed $m \in \mathbb{N}$, the mappings $T_{n}: X \rightarrow X$ and $f: X \rightarrow X$ satisfy $T_{n}^{m}(X) \subseteq f(X)$ for all $n \in \mathbb{N}$;

- $\quad$ the mappings $f$ and $T_{n}$ commute, i.e.,

$$
f\left(T_{n}(x)\right)=T_{n}(f(x))
$$

for all $x \in X$ and all $n \in \mathbb{N}$;

- the following contractive inequality is satisfied

$$
M\left(T_{i}^{m}(x), T_{j}^{m}(y), k_{i j} \cdot t\right) \geq M(f(x), f(y), t),
$$

where $k_{i j}$ satisfies $0<k_{i j} \leq k<1$ for all $i, j \in \mathbb{N}$ and for some constant $k$.

Then we have the following results.

(i) Suppose that the following conditions are satisfied:

- there exists $x^{*} \in X$ such that, given any $\lambda \in(0,1)$, there also exists $t_{\lambda}>0$ satisfying

$$
M\left(f\left(x^{*}\right), T_{1}^{m}\left(x^{*}\right), t_{\lambda}\right)>1-\lambda \text { and } \sup _{\lambda \in(0,1)} t_{\lambda}<+\infty .
$$

- $\quad$ any one of the following conditions is satisfied:

(a) $\quad(X, M)$ is $(<, \triangleright)$-complete and $f$ is simultaneously $(\triangleright, \triangleright)$-continuous and $(\triangleright, \triangleleft)$-continuous with respect to $M$;

(b) $\quad(X, M)$ is $(<, \triangleleft)$-complete and $f$ is simultaneously $(\triangleleft, \triangleright)$-continuous and $(\triangleleft, \triangleleft)$-continuous with respect to $M$.

Then the mappings $\left\{T_{n}^{m}\right\}_{n=1}^{\infty}$ and $f$ have a common coincidence point $x^{\circ}$. Moreover, the point $x^{\circ}$ can be obtained as follows:

- if condition (a) is satisfied, then $x^{\circ}$ can be obtained by taking the limit $f\left(x_{n}\right) \stackrel{M^{\triangleright}}{\longrightarrow} x^{\circ}$;

- if condition (b) is satisfied, then $x^{\circ}$ can be obtained by taking the limit $f\left(x_{n}\right) \stackrel{M^{\triangleleft}}{\longrightarrow} x^{\circ}$,

where the sequence $\left\{x_{n}\right\}_{n=1}^{\infty}$ is generated from the initial element $x_{0}=x^{*}$ according to (20).

(ii) Suppose that the following conditions are satisfied:

- there exists $x^{*} \in X$ such that, given any $\lambda \in(0,1)$, there exists $t_{\lambda}>0$ satisfying

$$
M\left(T_{1}^{m}\left(x^{*}\right), f\left(x^{*}\right), t_{\lambda}\right)>1-\lambda \text { and } \sup _{\lambda \in(0,1)} t_{\lambda}<+\infty
$$

- any one of the following conditions is satisfied: 
(c) $\quad(X, M)$ is $(>, \triangleright)$-complete and $f$ is simultaneously $(\triangleright, \triangleright)$-continuous and $(\triangleright, \triangleleft)$-continuous with respect to $M$;

(d) $\quad(X, M)$ is $(>, \triangleleft)$-complete and $f$ is simultaneously $(\triangleleft, \triangleright)$-continuous and $(\triangleleft, \triangleleft)$-continuous with respect to $M$.

Then the mappings $\left\{T_{n}^{m}\right\}_{n=1}^{\infty}$ and $f$ have a common coincidence point $x^{\circ}$. Moreover, the point $x^{\circ}$ can be obtained as follows:

- $\quad$ if condition (c) is satisfied, then $x^{\circ}$ can be obtained by taking the limit $f\left(x_{n}\right) \stackrel{M^{\triangleright}}{\longrightarrow} x^{\circ}$;

- $\quad$ if condition (d) is satisfied, then $x^{\circ}$ can be obtained by taking the limit $f\left(x_{n}\right) \stackrel{M^{\triangleleft}}{\longrightarrow} x^{\circ}$,

where the sequence $\left\{x_{n}\right\}_{n=1}^{\infty}$ is generated from the initial element $x_{0}=x^{*}$ according to (20).

Proof. We can construct the sequence $\left\{x_{n}\right\}_{n=1}^{\infty}$ from the initial element $x_{0}=x^{*}$ according to (20). Then we have $f\left(x^{*}\right)=f\left(x_{0}\right)$ and $T_{1}^{m}\left(x^{*}\right)=T_{1}^{m}\left(x_{0}\right)=f\left(x_{1}\right)$. From (20) and (22), we obtain

$$
M\left(f\left(x_{1}\right), f\left(x_{2}\right), t\right)=M\left(T_{1}^{m}\left(x_{0}\right), T_{2}^{m}\left(x_{1}\right), t\right) \geq M\left(f\left(x_{0}\right), f\left(x_{1}\right), \frac{t}{k_{12}}\right)
$$

and

$$
\begin{aligned}
M\left(f\left(x_{2}\right), f\left(x_{3}\right), t\right) & =M\left(T_{2}^{m}\left(x_{1}\right), T_{3}^{m}\left(x_{2}\right), t\right) \\
& \geq M\left(f\left(x_{1}\right), f\left(x_{2}\right), \frac{t}{k_{23}}\right) \geq M\left(f\left(x_{0}\right), f\left(x_{1}\right), \frac{t}{k_{12} \cdot k_{23}}\right) .
\end{aligned}
$$

By induction, we can obtain

$$
M\left(f\left(x_{n}\right), f\left(x_{n+1}\right), t\right) \geq M\left(f\left(x_{0}\right), f\left(x_{1}\right), \frac{t}{\prod_{i=1}^{n} k_{i, i+1}}\right) .
$$

Since the mapping $M(x, y, \cdot)$ is nondecreasing by Proposition 2 and $k_{i, i+1} \leq k$ for each $i \in \mathbb{N}$, from (25), we also have

$$
M\left(f\left(x_{n}\right), f\left(x_{n+1}\right), t\right) \geq M\left(f\left(x_{0}\right), f\left(x_{1}\right), \frac{t}{\prod_{i=1}^{n} k_{i, i+1}}\right) \geq M\left(f\left(x_{0}\right), f\left(x_{1}\right), \frac{t}{k^{n}}\right) .
$$

We can similarly obtain

$$
M\left(f\left(x_{n+1}\right), f\left(x_{n}\right), t\right) \geq M\left(f\left(x_{1}\right), f\left(x_{0}\right), \frac{t}{k^{n}}\right) .
$$

We consider the following cases.

- If (23) is satisfied, then, using (26) and part (i) of Proposition 13, we see that $\left\{f\left(x_{n}\right)\right\}_{n=1}^{\infty}$ is a $<$-Cauchy sequence in metric sense. If $(X, M)$ is $(<, \triangleright)$-complete or $(X, M)$ is $(<, \triangleleft)$-complete, then there exists $x^{\circ} \in X$ such that

$$
f\left(x_{n}\right) \stackrel{M^{\triangleright}}{\longrightarrow} x^{\circ} \text { or } f\left(x_{n}\right) \stackrel{M^{\triangleleft}}{\longrightarrow} x^{\circ},
$$

respectively, as $n \rightarrow \infty$.

- If (24) is satisfied, then, using (27) and part (ii) of Proposition 13, we see that $\left\{f\left(x_{n}\right)\right\}_{n=1}^{\infty}$ is a $>$-Cauchy sequence in metric sense. If $(X, M)$ is $(>, \triangleright)$-complete or $(X, M)$ is $(>, \triangleleft)$-complete, then there exists $x^{\circ} \in X$ such that (28) is satisfied. 
By the commutativity, we have

$$
f\left(f\left(x_{n}\right)\right)=f\left(T_{n}^{m}\left(x_{n-1}\right)\right)=T_{n}^{m}\left(f\left(x_{n-1}\right)\right)
$$

For any fixed mapping $T_{n}$, it follows that

$$
\begin{aligned}
M\left(f\left(f\left(x_{n+1}\right)\right), T_{n}^{m}\left(x^{\circ}\right), t\right) & =M\left(T_{n}^{m}\left(f\left(x_{n}\right)\right), T_{n}^{m}\left(x^{\circ}\right), t\right)(\text { by (29)) } \\
& \geq M\left(f\left(f\left(x_{n}\right)\right), f\left(x^{\circ}\right), \frac{t}{k_{n n}}\right)(\text { by (22)) } \\
& \geq M\left(f\left(f\left(x_{n}\right)\right), f\left(x^{\circ}\right), \frac{t}{k}\right)
\end{aligned}
$$

and

$$
M\left(T_{n}^{m}\left(x^{\circ}\right), f\left(f\left(x_{n+1}\right)\right), t\right)=M\left(T_{n}^{m}\left(x^{\circ}\right), T_{n}^{m}\left(f\left(x_{n}\right)\right), t\right) \geq M\left(f\left(x^{\circ}\right), f\left(f\left(x_{n}\right)\right), \frac{t}{k}\right) .
$$

To prove part (i) of this proposition, we consider the following cases.

- Suppose that $(X, M)$ is $(<, \triangleright)$-complete, and that $f$ is simultaneously $(\triangleright, \triangleright)$-continuous and $(\triangleright, \triangleleft)$-continuous with respect to M. From (28) and Proposition 3, we have

$$
\lim _{n \rightarrow \infty} M\left(f\left(f\left(x_{n}\right)\right), f\left(x^{\circ}\right), t\right)=1 \text {, i.e., } M\left(f\left(f\left(x_{n}\right)\right), f\left(x^{\circ}\right), t\right) \rightarrow 1-
$$

and

$$
\lim _{n \rightarrow \infty} M\left(f\left(x^{\circ}\right), f\left(f\left(x_{n}\right)\right), t\right)=1 \text {, i.e., } M\left(f\left(x^{\circ}\right), f\left(f\left(x_{n}\right)\right), t\right) \rightarrow 1-.
$$

Therefore, from (30) and (32), we obtain

$$
\begin{aligned}
1 & \geq \limsup _{n \rightarrow \infty} M\left(f\left(f\left(x_{n+1}\right)\right), T_{n}^{m}\left(x^{\circ}\right), t\right) \geq \liminf _{n \rightarrow \infty} M\left(f\left(f\left(x_{n+1}\right)\right), T_{n}^{m}\left(x^{\circ}\right), t\right) \\
& \geq \liminf _{n \rightarrow \infty} M\left(f\left(f\left(x_{n}\right)\right), f\left(x^{\circ}\right), \frac{t}{k}\right)=1,
\end{aligned}
$$

which says that

$$
\lim _{n \rightarrow \infty} M\left(f\left(f\left(x_{n+1}\right)\right), T_{n}^{m}\left(x^{\circ}\right), t\right)=1 \text {, i.e., } M\left(f\left(f\left(x_{n+1}\right)\right), T_{n}^{m}\left(x^{\circ}\right), t\right) \rightarrow 1-.
$$

Using the $\bowtie$-triangle inequality, we see that

$$
M\left(f\left(x^{\circ}\right), T_{n}^{m}\left(x^{\circ}\right), 2 t\right) \geq M\left(f\left(x^{\circ}\right), f\left(f\left(x_{n+1}\right)\right), t\right) * M\left(f\left(f\left(x_{n+1}\right)\right), T_{n}^{m}\left(x^{\circ}\right), t\right) .
$$

Using the left-continuity of t-norm $*$ and (33) and (34), we obtain $M\left(f\left(x^{\circ}\right), T_{n}^{m}\left(x^{\circ}\right), 2 t\right)=1$ for all $t>0$. Therefore we must have

$$
f\left(x^{\circ}\right)=T_{n}^{m}\left(x^{\circ}\right) .
$$

- Suppose that $(X, M)$ is $(<, \triangleleft)$-complete, and that $f$ is simultaneously $(\triangleleft, \triangleright)$-continuous and $(\triangleleft, \triangleleft)$-continuous with respect to $M$. Then the above argument is still valid to obtain $f\left(x^{\circ}\right)=T_{n}^{m}\left(x^{\circ}\right)$.

Therefore the mappings $\left\{T_{n}^{m}\right\}_{n=1}^{\infty}$ and $f$ have a common coincidence point $x^{\circ}$. Part (ii) of this proposition can be similarly obtained. This completes the proof.

Remark 3. Suppose that $f\left(x^{*}\right)$ and $T_{1}^{m}\left(x^{*}\right)$ have the finite distance beginning from $f\left(x^{*}\right)$ to $T_{1}^{m}\left(x^{*}\right)$. By definition, there exists $t^{*}<+\infty$ such that $M\left(f\left(x^{*}\right), T_{1}^{m}\left(x^{*}\right), t^{*}\right)=1$. If we take $t_{\lambda}=t^{*}$ for all $\lambda \in(0,1)$, then (23) is satisfied. 


\section{Common Fixed Points}

Let $X$ be a nonempty universal set. Recall that $x$ is called a common fixed point of $T$ and $f$ if and only if $x=f(x)=T(x)$. It is clear that the common fixed points are also the common coincidence points. Since the uniqueness of common coincidence points was not guaranteed, in this section, we shall provide the different arguments to prove the uniqueness of common fixed points. The uniqueness will be studied separately by assuming that $M$ satisfies the canonical condition and rational condition.

\subsection{Fuzzy Semi-Metric Space Satisfying the Canonical Condition}

We first investigate the uniqueness of common fixed points by assuming that the fuzzy semi-metric space $(X, M)$ satisfies the canonical condition.

Theorem 2. Let $(X, M)$ be a fuzzy semi-metric space along with a $t$-norm $*$ such that $M$ satisfies the canonical condition and the $\bowtie$-triangle inequality. Suppose that the following conditions are satisfied:

- the t-norm is left-continuous at 1 in the first or second component;

- for any fixed $x, y \in X$, the mapping $M(x, y, \cdot):(0, \infty) \rightarrow[0,1]$ is left-continuous at each point $t \in(0, \infty)$;

- given any fixed $m \in \mathbb{N}$, the mappings $T_{n}: X \rightarrow X$ and $f: X \rightarrow X$ satisfy $T_{n}^{m}(X) \subseteq f(X)$ for all $n \in \mathbb{N}$.

- the mappings $f$ and $T_{n}$ commute, i.e.,

$$
f\left(T_{n}(x)\right)=T_{n}(f(x))
$$

for all $x \in X$ and all $n \in \mathbb{N}$;

- $\quad$ the following contractive inequality is satisfied

$$
M\left(T_{i}^{m}(x), T_{j}^{m}(y), k_{i j} \cdot t\right) \geq M(f(x), f(y), t),
$$

where $k_{i j}$ satisfies $0<k_{i j} \leq k<1$ for all $i, j=1,2, \cdots$ and for some constant $k$.

Then we have the following results.

(i) Suppose that there exists $x^{*} \in X$ satisfying

$$
\sup _{\lambda \in(0,1)} \Gamma^{\downarrow}\left(\lambda, f\left(x^{*}\right), T_{1}^{m}\left(x^{*}\right)\right)<+\infty,
$$

and that any one of the following conditions is satisfied:

(a) $\quad(X, M)$ is $(<, \triangleright)$-complete and $f$ is simultaneously $(\triangleright, \triangleright)$-continuous and $(\triangleright, \triangleleft)$-continuous with respect to $M$;

(b) $\quad(X, M)$ is $(<, \triangleleft)$-complete and $f$ is simultaneously $(\triangleleft, \triangleright)$-continuous and $(\triangleleft, \triangleleft)$-continuous with respect to $M$.

Then the mappings $\left\{T_{n}^{m}\right\}_{n=1}^{\infty}$ and $f$ have a unique common fixed point $x^{\circ}$. Moreover, the point $x^{\circ}$ can be obtained as follows.

- If condition (a) is satisfied, then $x^{\circ}$ can be obtained by taking the limit $f\left(x_{n}\right) \stackrel{M^{\triangleright}}{\longrightarrow} x^{\circ}$;

- If condition (b) is satisfied, then $x^{\circ}$ can be obtained by taking the limit $f\left(x_{n}\right) \stackrel{M^{\triangleleft}}{\longrightarrow} x^{\circ}$,

where the sequence $\left\{x_{n}\right\}_{n=1}^{\infty}$ is generated from the initial element $x_{0}=x^{*}$ according to (20).

(ii) Suppose that there exists $x^{*} \in X$ satisfying

$$
\sup _{\lambda \in(0,1)} \Gamma^{\downarrow}\left(\lambda, T_{1}^{m}\left(x^{*}\right), f\left(x^{*}\right)\right)<+\infty,
$$


and that any one of the following conditions is satisfied:

(c) $\quad(X, M)$ is $(>, \triangleright)$-complete and $f$ is simultaneously $(\triangleright, \triangleright)$-continuous and $(\triangleright, \triangleleft)$-continuous with respect to $M$;

(d) $\quad(X, M)$ is $(>, \triangleleft)$-complete and $f$ is simultaneously $(\triangleleft, \triangleright)$-continuous and $(\triangleleft, \triangleleft)$-continuous with respect to $M$.

Then the mappings $\left\{T_{n}^{m}\right\}_{n=1}^{\infty}$ and $f$ have a unique common fixed point $x^{\circ}$. Moreover, the point $x^{\circ}$ can be obtained as follows.

- If condition (c) is satisfied, then $x^{\circ}$ can be obtained by taking the limit $f\left(x_{n}\right) \stackrel{M^{\triangleright}}{\longrightarrow} x^{\circ}$;

- If condition (d) is satisfied, then $x^{\circ}$ can be obtained by taking the limit $f\left(x_{n}\right) \stackrel{M^{\triangleleft}}{\longrightarrow} x^{\circ}$,

where the sequence $\left\{x_{n}\right\}_{n=1}^{\infty}$ is generated from the initial element $x_{0}=x^{*}$ according to (20).

Proof. We can construct the sequence $\left\{x_{n}\right\}_{n=1}^{\infty}$ from the initial element $x_{0}=x^{*}$ according to (20). Then we have $f\left(x^{*}\right)=f\left(x_{0}\right)$ and $T_{1}^{m}\left(x^{*}\right)=T_{1}^{m}\left(x_{0}\right)=f\left(x_{1}\right)$. We can similarly have (26) and (27).

- If (36) is satisfied, then, using (26) and Proposition 12, we see that $\left\{f\left(x_{n}\right)\right\}_{n=1}^{\infty}$ is a $<$-Cauchy sequence in metric sense. If $(X, M)$ is $(<, \triangleright)$-complete or $(X, M)$ is $(<, \triangleleft)$-complete, then there exists $x^{\circ} \in X$ such that (28) is satisfied.

- If (37) is satisfied, then, using (27) and Proposition 12, we see that $\left\{f\left(x_{n}\right)\right\}_{n=1}^{\infty}$ is a $>$-Cauchy sequence in metric sense. If $(X, M)$ is $(>, \triangleright)$-complete or $(X, M)$ is $(>, \triangleleft)$-complete, then there exists $x^{\circ} \in X$ such that (28) is satisfied.

Using the argument of the proof of Proposition 1, we can show that the mappings $\left\{T_{n}^{m}\right\}_{n=1}^{\infty}$ and $f$ have a common coincidence point $x^{\circ}$, i.e., $f\left(x^{\circ}\right)=T_{n}^{m}\left(x^{\circ}\right)$ for all $n \in \mathbb{N}$. Now we shall show that $x^{\circ}$ is a fixed point of $f$. Using (20), (22) and the nondecreasing property of $M(x, y, \cdot)$ by Proposition 2, we have

$$
\begin{aligned}
M\left(f\left(x_{n+1}\right), f\left(x^{\circ}\right), t\right) & =M\left(T_{n+1}^{m}\left(x_{n}\right), T_{n+1}^{m}\left(x^{\circ}\right), t\right) \\
& \geq M\left(f\left(x_{n}\right), f\left(x^{\circ}\right), \frac{t}{k_{n+1, n+1}}\right) \geq M\left(f\left(x_{n}\right), f\left(x^{\circ}\right), \frac{t}{k}\right) .
\end{aligned}
$$

and

$$
M\left(f\left(x^{\circ}\right), f\left(x_{n+1}\right), t\right)=M\left(T_{n+1}^{m}\left(x^{\circ}\right), T_{n+1}^{m}\left(x_{n}\right), t\right) \geq M\left(f\left(x^{\circ}\right), f\left(x_{n}\right), \frac{t}{k}\right) .
$$

By induction, we have

$$
M\left(f\left(x_{n+1}\right), f\left(x^{\circ}\right), t\right) \geq M\left(f\left(x_{1}\right), f\left(x^{\circ}\right), \frac{t}{k^{n}}\right)
$$

and

$$
M\left(f\left(x^{\circ}\right), f\left(x_{n+1}\right), t\right) \geq M\left(f\left(x^{\circ}\right), f\left(x_{1}\right), \frac{t}{k^{n}}\right) .
$$

Since $M$ satisfies the canonical condition, we have

$$
\lim _{t \rightarrow+\infty} M(x, y, t)=1 \text { for all } x, y \in X
$$


Since $0<k<1$, the inequalities (38) and (40) say that

$$
\begin{aligned}
1 & \geq \limsup _{n \rightarrow \infty} M\left(f\left(x_{n+1}\right), f\left(x^{\circ}\right), t\right) \geq \liminf _{n \rightarrow \infty} M\left(f\left(x_{n+1}\right), f\left(x^{\circ}\right), t\right) \\
& \geq \liminf _{n \rightarrow \infty} M\left(f\left(x_{1}\right), f\left(x^{\circ}\right), \frac{t}{k^{n}}\right)=1,
\end{aligned}
$$

which implies

$$
\lim _{n \rightarrow \infty} M\left(f\left(x_{n+1}\right), f\left(x^{\circ}\right), t\right)=1 .
$$

Using (39), we can similarly obtain

$$
\lim _{n \rightarrow \infty} M\left(f\left(x^{\circ}\right), f\left(x_{n+1}\right), t\right)=1 .
$$

To prove part (i) of this proposition, since $(X, M)$ is $(<, \triangleright)$-complete or $(X, M)$ is $(<, \triangleleft)$-complete, by referring to (28), there exists $x^{\circ} \in X$ such that $f\left(x_{n}\right) \stackrel{M^{\triangleright}}{\longrightarrow} x^{\circ}$ or $f\left(x_{n}\right) \stackrel{M^{\triangleleft}}{\longrightarrow} x^{\circ}$, respectively, as $n \rightarrow \infty$.

- If $f\left(x_{n}\right) \stackrel{M^{\triangleright}}{\longrightarrow} x^{\circ}$, i.e.,

$$
\lim _{n \rightarrow \infty} M\left(f\left(x_{n+1}\right), x^{\circ}, t\right)=1,
$$

then the $\bowtie$-triangle inequality says that

$$
M\left(f\left(x^{\circ}\right), x^{\circ}, 2 t\right) \geq M\left(f\left(x^{\circ}\right), f\left(x_{n+1}\right), t\right) * M\left(f\left(x_{n+1}\right), x^{\circ}, t\right) .
$$

Using the left-continuity of t-norm $*$ and (42) and (43), we can obtain $M\left(f\left(x^{\circ}\right), x^{\circ}, 2 t\right)=1$ for all $t>0$ by taking $n \rightarrow \infty$.

- If $f\left(x_{n}\right) \stackrel{M^{\triangleleft}}{\longrightarrow} x^{\circ}$, i.e.,

$$
\lim _{n \rightarrow \infty} M\left(x^{\circ}, f\left(x_{n+1}\right), t\right)=1 .
$$

then the $\bowtie$-triangle inequality says that

$$
M\left(x^{\circ}, f\left(x^{\circ}\right), 2 t\right) \geq M\left(x^{\circ}, f\left(x_{n+1}\right), t\right) * M\left(f\left(x_{n+1}\right), f\left(x^{\circ}\right), t\right) .
$$

Using the left-continuity of t-norm $*$ and (41) and (44), we can obtain $M\left(x^{\circ}, f\left(x^{\circ}\right), 2 t\right)=1$ for all $t>0$ by taking $n \rightarrow \infty$.

The above two cases show that $x^{\circ}=f\left(x^{\circ}\right)$.

Let $\bar{x}$ be another point in $X$ satisfying $f(\bar{x})=T_{n}^{m}(\bar{x})$ for all $n \in \mathbb{N}$. By (22) and the nondecreasing property of $M(x, y, \cdot)$, we have

$$
M\left(f\left(x^{\circ}\right), f(\bar{x}), t\right)=M\left(T_{n}^{m}\left(x^{\circ}\right), T_{n}^{m}(\bar{x}), t\right) \geq M\left(f\left(x^{\circ}\right), f(\bar{x}), \frac{t}{k_{n n}}\right) \geq M\left(f\left(x^{\circ}\right), f(\bar{x}), \frac{t}{k}\right) .
$$

By induction, we can obtain

$$
M\left(f\left(x^{\circ}\right), f(\bar{x}), t\right) \geq M\left(f\left(x^{\circ}\right), f(\bar{x}), \frac{t}{k^{n}}\right) .
$$

Using the canonical condition, we obtain $M\left(f\left(x^{\circ}\right), f(\bar{x}), t\right)=1$ for all $t>0$, i.e.,

$$
f\left(x^{\circ}\right)=f(\bar{x}) .
$$

Now, for each $n \in \mathbb{N}$, since

$$
f\left(T_{n}\left(x^{\circ}\right)\right)=T_{n}\left(f\left(x^{\circ}\right)\right)=T_{n}\left(T_{n}^{m}\left(x^{\circ}\right)\right)=T_{n}^{m}\left(T_{n}\left(x^{\circ}\right)\right)
$$


By regarding $\bar{x}$ as $T_{n}\left(x^{\circ}\right)$, the equality (47) says that $f(\bar{x})=T_{n}^{m}(\bar{x})$. Therefore, according to (46), we must have

$$
f\left(x^{\circ}\right)=f(\bar{x})=f\left(T_{n}\left(x^{\circ}\right)\right)=T_{n}\left(f\left(x^{\circ}\right)\right),
$$

which implies $x^{\circ}=T_{n}\left(x^{\circ}\right)$, since $x^{\circ}=f\left(x^{\circ}\right)$. This shows that $x^{\circ}$ is a common fixed point of $\left\{T_{n}\right\}_{n \in \mathbb{N}}$ and $f$.

To prove the uniqueness, let $\widehat{x}$ be another common fixed point of $\left\{T_{n}\right\}_{n \in \mathbb{N}}$ and $f$, i.e., $\widehat{x}=f(\widehat{x})=T_{n}(\widehat{x})$ for all $n \in \mathbb{N}$. Then

$$
f(\widehat{x})=T_{n}(\widehat{x})=T_{n}(f(\widehat{x}))=T_{n}^{2}(\widehat{x})=\cdots=T_{n}^{m}(\widehat{x}) .
$$

Therefore, by referring to the derivation of (46), we can obtain

$$
x^{\circ}=f\left(x^{\circ}\right)=f(\widehat{x})=\widehat{x} .
$$

Part (ii) of this proposition can be similarly obtained, and the proof is complete.

\subsection{Fuzzy Semi-Metric Space Satisfying the Rational Condition}

Now we investigate the uniqueness of common fixed points by assuming that the fuzzy semi-metric space $(X, M)$ satisfies the rational condition.

Theorem 3. Let $(X, M)$ be a fuzzy semi-metric space along with a t-norm $*$. Suppose that $M$ satisfies the rational condition and the $\bowtie$-triangle inequality, and that the following conditions are satisfied:

- $\quad M$ satisfies the distant condition in Definition 5.

- $\quad$ the following inequality is satisfied:

$$
\sup _{n}\left(a_{n} * b_{n}\right) \geq\left(\sup _{n} a_{n}\right) *\left(\sup _{n} b_{n}\right)
$$

for any sequences $\left\{a_{n}\right\}_{n=1}^{\infty}$ and $\left\{b_{n}\right\}_{n=1}^{\infty}$ in $[0,1]$.

- the t-norm is left-continuous at 1 in the first or second component;

- for any fixed $x, y \in X$, the mapping $M(x, y, \cdot):(0, \infty) \rightarrow[0,1]$ is continuous on $(0, \infty)$;

- $\quad$ given any fixed $m \in \mathbb{N}$, the mappings $T_{n}: X \rightarrow X$ and $f: X \rightarrow X$ satisfy $T_{n}^{m}(X) \subseteq f(X)$ for all $n \in \mathbb{N}$.

- the mappings $f$ and $T_{n}$ commute, i.e.,

$$
f\left(T_{n}(x)\right)=T_{n}(f(x))
$$

for all $x \in X$ and all $n \in \mathbb{N}$;

- the following contractive inequality is satisfied

$$
M\left(T_{i}^{m}(x), T_{j}^{m}(y), k_{i j} \cdot t\right) \geq M(f(x), f(y), t)
$$

where $k_{i j}$ satisfies $0<k_{i j} \leq k<1$ for all $i, j=1,2, \cdots$ and for some constant $k$.

- $\quad$ any one of the following conditions is satisfied:

- $\quad$ the mapping $f$ is simultaneously $(\triangleright, \triangleright)$-continuous and $(\triangleright, \triangleleft)$-continuous with respect to $M$;

- $\quad$ the mapping $f$ is simultaneously $(\triangleleft, \triangleright)$-continuous and $(\triangleleft, \triangleleft)$-continuous with respect to $M$.

(i) Assume that $(X, M)$ is $(<, \triangleright)$-complete and $(<, \triangleleft)$-complete, and that there exists $x^{*} \in X$ such that, given any $\lambda \in(0,1)$, there exists $t_{\lambda}>0$ satisfying 


$$
M\left(f\left(x^{*}\right), T_{1}^{m}\left(x^{*}\right), t_{\lambda}\right)>1-\lambda \text { and } \sup _{\lambda \in(0,1)} t_{\lambda}<+\infty .
$$

Then the mappings $\left\{T_{n}^{m}\right\}_{n=1}^{\infty}$ and $f$ have a unique common fixed point $x^{\circ}$.

(ii) Assume that $(X, M)$ is $(>, \triangleright)$-complete and $(>, \triangleleft)$-complete, and that there exists $x^{*} \in X$ such that, given any $\lambda \in(0,1)$, there exists $t_{\lambda}>0$ satisfying

$$
M\left(T_{1}^{m}\left(x^{*}\right), f\left(x^{*}\right), t_{\lambda}\right)>1-\lambda \text { and } \sup _{\lambda \in(0,1)} t_{\lambda}<+\infty .
$$

Then the mappings $\left\{T_{n}^{m}\right\}_{n=1}^{\infty}$ and $f$ have a unique common fixed point $x^{\circ}$.

Moreover, the point $x^{\circ}$ as shown above can be obtained by taking the limit $f\left(x_{n}\right) \stackrel{M^{\triangleright}}{\longrightarrow} x^{\circ}$ or the limit $f\left(x_{n}\right) \stackrel{M^{\triangleleft}}{\longrightarrow} x^{\circ}$, where the sequence $\left\{x_{n}\right\}_{n=1}^{\infty}$ is generated from the initial element $x_{0}=x^{*}$ according to (20).

Proof. To prove part (i) of this proposition, we first have (26) in Theorem 1. Using (48), (26) and part (i) of Proposition 13 by taking the initial element $x_{0}=x^{*}$ (i.e., $f\left(x^{*}\right)=f\left(x_{0}\right)$ and $T_{1}^{m}\left(x^{*}\right)=$ $\left.T_{1}^{m}\left(x_{0}\right)=f\left(x_{1}\right)\right)$, we see that $\left\{f\left(x_{n}\right)\right\}_{n=1}^{\infty}$ is a $<$-Cauchy sequence. Since $(X, M)$ is $(<, \triangleright)$-complete and $(<, \triangleleft)$-complete, from Proposition 3 , there exists $x^{\circ} \in X$ such that $f\left(x_{n}\right) \stackrel{M^{\triangleright}}{\longrightarrow} x^{\circ}$ and $f\left(x_{n}\right) \stackrel{M^{\triangleleft}}{\longrightarrow} x^{\circ}$ as $n \rightarrow \infty$, which also says that $f\left(x_{n}\right) \stackrel{M}{\longrightarrow} x^{\circ}$ as $n \rightarrow \infty$. The similar argument from the proof of Theorem 1 shows that $T_{n}^{m}\left(x^{\circ}\right)=f\left(x^{\circ}\right)$ for all $n \in \mathbb{N}$. Now, we shall show that $x^{\circ}$ is a fixed point of $f$. Using (20), (22) and the nondecreasing property of $M(x, y, \cdot)$ by Proposition 2, we have

$$
\begin{aligned}
M\left(f\left(x_{n+1}\right), f\left(x^{\circ}\right), t\right)= & M\left(T_{n}^{m}\left(x_{n}\right), T_{n}^{m}\left(x^{\circ}\right), t\right) \\
& \geq M\left(f\left(x_{n}\right), f\left(x^{\circ}\right), \frac{t}{k_{n n}}\right) \geq M\left(f\left(x_{n}\right), f\left(x^{\circ}\right), \frac{t}{k}\right)
\end{aligned}
$$

Since $f\left(x_{n}\right) \stackrel{M}{\longrightarrow} x^{\circ}$ as $n \rightarrow \infty$, applying Proposition 4 to (50), we obtain

$$
M\left(x^{\circ}, f\left(x^{\circ}\right), t\right) \geq M\left(x^{\circ}, f\left(x^{\circ}\right), \frac{t}{k}\right) .
$$

By induction, we can obtain

$$
M\left(x^{\circ}, f\left(x^{\circ}\right), t\right) \geq M\left(x^{\circ}, f\left(x^{\circ}\right), \frac{t}{k^{n}}\right),
$$

which says that

$$
M\left(x^{\circ}, f\left(x^{\circ}\right), k^{n} \cdot t\right) \geq M\left(x^{\circ}, f\left(x^{\circ}\right), t\right) \geq 0 .
$$

Since $M$ satisfies the rational condition, for $x \neq y$, we have

$$
\lim _{t \rightarrow 0+} M(x, y, t)=0 .
$$

Suppose that $x^{\circ} \neq f\left(x^{\circ}\right)$. Since $0<k<1$, by applying (52) to (51), it follows that $M\left(x^{\circ}, f\left(x^{\circ}\right), t\right)=0$ for all $t>0$ by taking $n \rightarrow \infty$. However, the distant condition says that there exists $t_{0}>0$ such that $M\left(x^{\circ}, f\left(x^{\circ}\right), t_{0}\right) \neq 0$. This contradiction shows that $x^{\circ}=f\left(x^{\circ}\right)$. The remaining proof follows from the argument of the proof of Theorem 2.

To prove part (ii) of this proposition, using (49) and part (ii) of Proposition 13 by taking the initial element $x_{0}=x^{*}$ (i.e., $f\left(x^{*}\right)=f\left(x_{0}\right)$ and $T_{1}^{m}\left(x^{*}\right)=T_{1}^{m}\left(x_{0}\right)=f\left(x_{1}\right)$ ), we see that $\left\{f\left(x_{n}\right)\right\}_{n=1}^{\infty}$ is a $>$-Cauchy sequence. Since $(X, M)$ is $(>, \triangleright)$-complete and $(>, \triangleleft)$-complete, from Proposition 3 , there exists $x^{\circ} \in X$ such that $f\left(x_{n}\right) \stackrel{M^{\triangleright}}{\longrightarrow} x^{\circ}$ and $f\left(x_{n}\right) \stackrel{M^{\triangleleft}}{\longrightarrow} x^{\circ}$ as $n \rightarrow \infty$, which also says that $f\left(x_{n}\right) \stackrel{M}{\longrightarrow} x^{\circ}$ as $n \rightarrow \infty$. The proof in part (i) of this proposition is still valid. This completes the proof. 
Conflicts of Interest: The author declares no conflict of interest.

\section{References}

1. Schweizer, B.; Sklar, A. Statistical Metric Spaces. Pac. J. Math. 1960, 10, 313-334.

2. Schweizer, B.; Sklar, A.; Thorp, E. The Metrization of Statistical Metric Spaces. Pac. J. Math. 1960, 10, 673-675.

3. Schweizer, B.; Sklar, A. Triangle Inequalities in a Class of Statistical Metric Spaces. J. Lond. Math. Soc. 1963, 38, 401-406.

4. Hadžić, O.; Pap, E. Fixed Point Theory in Probabilistic Metric Spaces; Klumer Academic Publishers: Dordrecht, The Netherlands, 2001.

5. Chang, S.S.; Cho, Y.J.; Kang, S.M. Nonlinear Operator Theory in Probabilistic Metric Space; Nova Science Publishers: New York, NY, USA, 2001.

6. Kramosil, I.; Michalek, J. Fuzzy Metric and Statistical Metric Spaces. Kybernetika 1975, 11, 336-344.

7. Singh, B.; Chauhan, M.S. Common Fixed Points of Compatible Maps in Fuzzy Metric Spaces. Fuzzy Sets Syst. 2000, 115, 471-475.

8. Vasuki, R. A Common Fixed Point Theorem in a Fuzzy Metric Space. Fuzzy Sets Syst. 1998, 97, $395-397$.

9. Wang, S.; Alsulami, S.M.; Cirić, L. Common fixed point theorems for nonlinear contractive mappings in fuzzy metric spaces. Fixed Point Theory Appl. 2013, 2013, 191.

10. De la Sen, M.; Foldán, A.F.; Agarwal, R.P. On Contrcative Cyclic Fuzzy Maps in Metric spaces and Some Related Results on Fuzzy Best Proximity Points and Fuzzy Fixed Points. Fixed Points Theory Appl. 2015, 2015, 103.

11. De la Sen, M.; Abbas, M.; Saleem, N. On Optimal Fuzzy Best Proximity Coincidence Points of Proximal Contractions Involving Cyclic Mappings in Non-Archimedean Fuzzy Metric Spaces. Mathematics 2017, 5, 22.

12. Wu, H.-C. Hausdorff Topology Induced by the Fuzzy Metric and the Fixed Point Theorems in Fuzzy Metric Spaces. J. Korean Math. Soc. 2015, 52, 1287-1303.

(C) 2018 by the authors. Licensee MDPI, Basel, Switzerland. This article is an open access article distributed under the terms and conditions of the Creative Commons Attribution (CC BY) license (http://creativecommons.org/licenses/by/4.0/). 